Cahiers

d'ethnomusicologie

\section{Cahiers d'ethnomusicologie}

Anciennement Cahiers de musiques traditionnelles

$7 \mid 1994$

Esthétiques

\title{
Harpes et harpistes du Haut-Oubangui
}

Paris : Université de Paris X, 1994

\section{Susanne Fürniß}

\section{(2) OpenEdition}

Journals

Édition électronique

URL : http://journals.openedition.org/ethnomusicologie/1454

ISSN : 2235-7688

Éditeur

ADEM - Ateliers d'ethnomusicologie

\section{Édition imprimée}

Date de publication : 31 décembre 1994

Pagination : 264-266

ISBN : 2-8257-0503-9

ISSN : $1662-372 X$

\section{Référence électronique}

Susanne Fürniß, « Harpes et harpistes du Haut-Oubangui », Cahiers d'ethnomusicologie [En ligne],

7 | 1994, mis en ligne le 03 janvier 2012, consulté le 05 mai 2019. URL : http://

journals.openedition.org/ethnomusicologie/1454

Ce document a été généré automatiquement le 5 mai 2019.

Tous droits réservés 


\title{
Harpes et harpistes du Haut- Oubangui
}

Paris : Université de Paris X, 1994

\author{
Susanne Fürniß
}

\section{RÉFÉRENCE}

Harpes et harpistes du Haut-Oubangui.Ouvrage collectif sous la responsabilité d'Eric de DAMPIERRE. Paris : Université de Paris X, Laboratoire d'ethnologie et de sociologie comparative, Mission sociologique du Haut-Oubangui, Collection « Ateliers » 14, 1994, $148 \mathrm{p}$.

1 Le dernier-né de la série de publications consacrées aux harpes zandé et nzakara nous donne enfin une idée de la systématique musicale avec «La musique de la harpe » de Marc Chemillier. Cet article important est précédé de trois contributions centrées autour de l'esthétique dans le contexte de la société traditionnelle zandé - «Harpes zandé : sculpter au singulier» (Gaetano Speranza), «Le contexte du souci esthétique (sculpture, rhétorique et musique) dans la civilisation zandé » (Eric de Dampierre) et « Sculpture sans sculpteur?» (Gaetano Speranza) - et suivi, en appendice, d'«Instructions au photographe, pour photographier décemment une harpe » (Eric de Dampierre). Aussi instructif que soient ces quatre articles pour la compréhension profonde de l'ensemble social harpe-harpiste-musique de harpe, je ne parlerai ici que du texte consacré à la musique.

2 A partir d'enregistrements et d'enquêtes effectués il y a 25 à 30 ans par Eric de Dampierre, Marc Chemillier tente de compléter, depuis 1989, les données tant ethnologiques que musicales. Contrairement au répertoire enregistré auprès des jeunes par Gerhard Kubik (1964), il s'agit ici d'un répertoire ancien qui n'avait pas encore été étudié de près. Si ce répertoire était déjà « vieux » il y a trente ans, on comprend facilement les problèmes qu'eut Chemillier à rassembler des musiciens encore compétents. 
3 A la manière de Vincent Dehoux (1986) et avec une honnêteté remarquable, l'auteur fait précéder son analyse musicale d'un compte rendu de mission qui illustre de façon éloquente les aléas du travail sur le terrain. La fragilité des traditions se manifeste dans la fiabilité variable de musiciens octogénaires et la disparition des instruments - « toutes les pièces que nous avons enregistrées ont été jouées sur une harpe que Eric de Dampierre avait fait construire en 1969, et que nous avons apportée nous-mêmes dans nos bagages » (76). Toutefois, dans un style captivant, l'auteur nous rappelle que, grâce à la patience et à la qualité des rapports personnels, ce travail parfois pénible s'est vu ponctué de moments forts tels le don d'une harpe ancestrale ou encore la réalisation de «mon projet le plus cher... reconstituer un orchestre complet de la danse ngbàkià » (86). La réunion, pour cette occasion, des rares musiciens initiés à ce répertoire, dispersés à travers tout le pays nzakara, se heurta violemment à la foi protestante de certains protagonistes convertis, et le problème ne fut résolu qu'après maintes négociations et l'intervention de l'autorité religieuse.

4 L'analyse du répertoire des harpistes prend son départ avec l'écoute des anciens enregistrements et l'identification des pièces. Leur appartenance aux différentes catégories musicales est déterminée, entre autre, à l'aide d'enregistrements inspirés du " re-recording». Il en résulte que "ces catégories musicales forment un système de classification pouvant s'appliquer à l'ensemble du répertoire des poètes-harpistes; puis que chaque catégorie correspond à une danse traditionnelle nzakara; et enfin que l'appartenance d'une pièce à une catégorie repose sur des critères proprement musicaux " (90). S'il est toujours agréable de relire ce que l'on a déjà appris à travers des études d'autres cultures, ce résultat met notamment en valeur le travail de l'auteur.

5 L'article présente deux points forts. Chemillier s'efforce à tout moment d'inclure dans l'analyse musicale toutes les informations ethnologiques, historiques et technologiques qu'il tient en mains. Ceci l'amène à mettre en évidence le prolongement du rapport social privilégié entre deux répertoires jusque dans leurs structures musicales respectives (120). De même, il explique de façon claire le rapport entre accords de harpe et de xylophone et nous fait découvrir une particularité tout à fait passionnante du premier : «il apparaît non moins certain que cet accord pentatonique anhémitonique s'oppose, dans le système d'échelles musicales nzakara, à d'autres accords très différents » (101). On regrette de ne pas les voir tous exposés...

$6 \quad$ Le second point concerne un aspect particulier d'une petite partie du corpus - les pièces des catégories ngbàkià et limanza, dont la période est constituée de cinq ou dix pulsations. Ces pièces révèlent une structure ergonomique en canon : la succession du doigté d'une main est reproduite de façon quasi identique de l'autre main et ce, à des distances temporelles variables selon les pièces. Ceci donne lieu à une répétition d'un même contour mélodique dans le grave, dont les trois degrés constitutifs sont transposés d'une tierce majeure, d'une quarte et d'un triton respectivement (117). La musique des deux répertoires précités - le premier lié à l'autorité, le second au pouvoir (120) - est d'autant plus intéressante qu'elle surprend par le nombre élevé de constructions mélodiques à partir de seulement cinq consonances acceptables. En effet, dans un contexte strictement homorythmique, un nouveau paramètre créateur de variantes musicales intervient : celui de la forte variabilité du cycle (114).

7 Cette étude n'est qu'un premier aperçu d'une vaste enquête ethnomusicologique. De là quelques défauts ou, plutôt, quelques frustrations. L'analyse très poussée de certains phénomènes rythmiques est souvent marquée par une confusion entre les notions de 
mètre et de rythme; de même, la terminologie relative aux notions de catégorie, de version, d'air, de pièce, de formule de référence ou de modèle reste parfois un peu floue.

Dans ses prospectives, Marc Chemillier lance le défi de résoudre une contradiction apparente. Il présente la musique de harpe nzakara comme étroitement liée à la danse, et le lecteur se demande comment cela se relie à l'affirmation de Dampierre selon laquelle les sons de la harpe « ont la délectation individuelle pour fin » et que « jouer de la harpe vise au Beau musical» (59) ? L'auteur se propose alors d'explorer notamment le rapport entre fonction sociale d'une danse et thème abordé dans les chants des harpistes, entreprise très difficile me semble-t-il, vu le caractère presque ésotérique de certains chants: «...Nzakara et Zandé sont passés maîtres, maîtres de la parole railleuse où l'allusion n'est jamais transparente et se voit sans cesse désamorcée « (Dampierre, 63).

Enfin, Chemillier opère un rapprochement entre structure musicale, esthétique et mode de penser nzakara afin d'expliquer l'apparition d'un phénomène musical proche du canon dans un contexte de musique de tradition orale. Ainsi, les Nzakara rejetteraient le concept d'identité, mais manifesteraient une tendance à grouper par paire des choses « presque identiques » (133). Des projets de recherche qui tiennent en haleine...

En attendant la suite des publications sur les harpes zandé - source apparemment intarissable -, on peut regretter que ce fascicule ne soit pas commercialisé ${ }^{1}$. En effet, par son approche diversifiée, il serait à recommander à toute personne s'intéressant à une culture musicale africaine.

\section{BIBLIOGRAPHIE}

DEHOUX Vincent, 1986, Chants à penser gbaya (Centrafrique). Paris : SELAF.

KUBIK Gerhard, 1964, « Harp music of the Azande and related people in the Central African Republic ». African Music III : 37-76.

\section{NOTES}

1. On peut obtenir cet ouvrage au prix de $20 \mathrm{FF}$ au secrétariat du Laboratoire d'Ethnologie de l'Université de Paris X-Nanterre, 200 Avenue de la République, 92100 Nanterre cedex. Une édition révisée est actuellement en préparation. 